\title{
PERFIL SOCIODEMOGRÁFICO DOS ESTUDANTES DE ENFERMAGEM DA UNIVERSIDADE ESTADUAL VALE DO ACARAÚ (UVA)
}

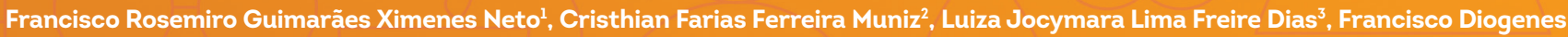
Júnior ${ }^{4}$, Maria Adelane Monteiro da Silva5, Eliany Nazaré Oliveira ${ }^{6}$

Objetivo: descrever o perfil sociodemográfico dos estudantes do curso de Graduação em Enfermagem da Universidade Estadual Vale do Acaraú (UVA). Metodologia: estudo exploratório-descritivo, sob abordagem quantitativa, realizado no período de novembro de 2015 a setembro de 2017, com 276 acadêmicos de Enfermagem da UVA. Os dados foram coletados por meio de questionário eletrônico através da plataforma Google Forms ${ }^{\circledR}$. Resultados: 78,6\% dos estudantes são do sexo feminino; $56,5 \%$ são adultos jovens com idade de 20 a 24 anos; 89,9\% são solteiros; 57,2\% de raça/cor parda; e 41,7\% com renda familiar mensal de até um salário mínimo. Conclusão: com o atual contexto social, político e educacional da Enfermagem e o do SUS, os dados desta pesquisa contribuirão para o aperfeiçoamento do projeto pedagógico do curso e de políticas públicas para essa que é uma das mais importantes categorias de trabalhadores da saúde.

Descritores: Enfermagem, Ensino em Enfermagem, Estudantes de Enfermagem.

\section{SOCIODEMOGRAPHIC PROFILE OF UNDERGRADUATE NURSING STUDENTS OF THE STATE UNIVERSITY VALE DO ACARAÚ (UVA)}

Objective: To describe the sociodemographic profile of undergraduate nursing students from the State University of Vale do Acaraú (UVA). Methodology: Exploratory-descriptive study, under a quantitative approach, conducted in the period from November 2015 to September 2017 with 276 UVA Nursing students. The data collection was done by an electronic questionnaire through the Google Forms ${ }^{\circledR}$ platform. Results: $78.6 \%$ of the students are female; $56.5 \%$ are young adults aged 20 to 24 years; $89.9 \%$ are single; $57.2 \%$ race / brown color; and $41.7 \%$ with monthly family income up to one minimum wage. Conclusion: With the current social, political and educational context of Nursing and SUS, the data from this research will contribute in the improvement of the educational project of the course and public policies for this, that is, one of the most important categories of health workers.

Descriptors: Nursing, Teaching in Nursing, Nursing students.

\section{PERFIL SOCIODEMOGRÁFICO DE LOS ESTUDIANTES DE ENFERMERÍA DE LA UNIVERSIDAD ESTADUAL VALLE DE ACARAÚ (UVA)}

Objetivo: Describir el perfil sociodemográfico de los estudiantes del curso de Graduación en Enfermería de la Universidad Estadual Valle de Acaraú (UVA). Metodología: Estudio exploratorio-descriptivo, con abordaje cuantitativo, realizado en el periodo de noviembre de 2015 a septiembre de 2017 con 276 académicos de Enfermería de la UVA. Los datos fueron recolectados por medio de cuestionario electrónico a través de la plataforma Google Forms ${ }^{\circledR}$. Resultados: 78,6\% de los estudiantes son de sexo femenino; $56,5 \%$ son adultos jóvenes con edad de 20 a 24 años; $89,9 \%$ son solteros; 57,2\% mulatos; y 41,7\% con renta familiar mensual de hasta un salario mínimo. Conclusión: Con el actual contexto social, político y educacional de la Enfermería y del SUS, los datos de esta investigación contribuirán al perfeccionamiento del proyecto pedagógico del curso y de políticas públicas para esta, que es una de las más importantes categorias de trabajadores de la salud.

Descriptores: Enfermería, Enseñanza en Enfermería, Estudiantes de Enfermería.

'Enfermeiro, Doutor em Ciências. Docente do Curso de Enfermagem e do Mestrado em Saúde da Familia da Universidade Estadual Vale do Acaraú (UVA),

Sobral - Ceará. E-mail: rosemironeto@gmail.com.

${ }^{2}$ Enfermeiro Graduado pela UVA. Hospital do Coração de Sobral - Ceará.

${ }^{3}$ Acadêmica de Enfermagem da UVA.

${ }^{4}$ Enfermeiro Graduado pela UVA. Hospital do Municipal Natércia Rios. Itarema - Ceará.

${ }^{5}$ Enfermeira, Doutor em Enfermagem. Docente e Coordenadora do Curso de Enfermagem e Docente do Mestrado em Saúde da Familia da UVA.

${ }^{6}$ Enfermeira, Doutor em Psicologia. Docente do Curso de Enfermagem e do Mestrado em Saúde da Familia da UVA. 


\section{INTRODUÇÃO}

Comoadvento do SistemaÚnico deSaúde(SUS), otrabalho em Enfermagem passa por um processo de ressignificação, com a aquisição de novas práticas e saberes, para dar conta das demandas, problemas e necessidades de saúde, tendo como foco a família, os sujeitos e sua comunidade, em uma perspectiva da concepção de saúde, como qualidade de vida. Com isso, as novas intervenções no campo da Saúde Coletiva e da Saúde Pública são cartografadas com o intuito de aperfeiçoar a atenção ao processo saúde-doença-cuidado.

Consequentemente, a reestruturação produtiva e a transição tecnológica do setor saúde vêm influindo em diversas transformações no ensino da Enfermagem no Brasil, na tentativa de formar enfermeiros generalistas, com uma visão sanitária voltada para as necessidades do sistema público sanitário. Uma importante estratégia para adequar o ensino de Enfermagem a esse panorama sanitário, fora as Diretrizes Curriculares Nacionais (DCN), que definem os princípios, fundamentos, condições e procedimentos da formação de enfermeiros com enfoque generalista, humanista, críticos e reflexivos ${ }^{(1)}$.

Com o estabelecimento das DCN, o perfil de formação e, consequentemente, o perfil profissional da Enfermagem brasileira vêm passando por intensas mudanças políticas, para adequarem-se ao cenário contemporâneo do mercado de trabalho em saúde. Tal padrão de formação tem como base o perfil epidemiológico regionalizado, as ações de prevenção de riscos, agravos e doenças, a educação e a promoção da saúde, dentre outras, e não somente na lógica tecnicista do modelo de ensino flexneriano, caracterizado por estar centrado apenas na doença.

Com o desenvolvimento do SUS e a implantação e/ou implementação de novas políticas, programas, projetos, ações e serviços de saúde, ocorreu um crescimento geométrico dos cursos de graduação em Enfermagem. No ano de 2000, havia 183 cursos de graduação em Enfermagem, entre instituições públicas e privadas; em 2006, eram 582 cursos, um crescimento de $218 \%$. Já no ano de 2015 , o Ministério da Educação contabilizou um total de 793 cursos, assim distribuidos entre todas as regiões do país: Região Sudeste - 329; Região Nordeste - 198; Região Sul - 112; Região CentroOeste - 84; e 70 na Região Norte. O estado do Ceará possui 32 cursos de Graduação em Enfermagem ${ }^{(2)}$.

A ampliação do número de vagas nos cursos de graduação em Enfermagem, em um determinado estágio de desenvolvimento do SUS, foi importantíssimo, pois a demanda de novos enfermeiros, com o perfil de formação profissional para o contexto político sanitário voltado a atender às necessidades do país, era urgente. Porém, o que temos hoje é um número excessivo de cursos de graduação em
Enfermagem, demandados pelo processo de mercantilização do ensino nas Ciências da Saúde, e que nos levar a questionar/ refletir de como está a qualidade desse ensino e com que competência estão sendo formados os novos enfermeiros.

Destarte, com a expansão dos cursos de Enfermagem nos últimos anos, com consequente aumento no número de acadêmicos matriculados, surgiu o interesse de se investigar o perfil do estudante do curso de graduação em Enfermagem da Universidade Estadual Vale do Acaraú (UVA), por ser uma Instituição de referência no norte cearense, que forma aproximadamente 70 graduados em Enfermagem por ano. Este estudo assume relevância para o ensino superior, na medida em que poderá subsidiar propostas de reestruturação de currículos de Enfermagem e propor adaptações, reforçando a coerência teórico-prática do processo de formação, de modo a complementar as necessidades pessoais e regionais e o perfil epidemiológico da população. Ademais, poderá contribuir efetivamente no processo ensino-aprendizagem nas instituições, no campo de estudo e na análise do crescimento da profissão na região, no estado do Ceará e mesmo no país. Ainda contribuirá com o curso de Enfermagem, uma vez que delineado o perfil desse público, se tornará mais fácil a aplicação de novas estratégias que visem a otimização do aprendizado e, consequentemente, a formação de um profissional competente, humanizado e com responsabilidade sanitária. Assim, o presente estudo objetivou descrever o perfil sociodemográfico dos estudantes do curso de graduação em Enfermagem da UVA.

\section{METODOLOGIA}

Estudo exploratório-descritivo, sob abordagem quantitativa, realizado no período de novembro de 2015 a setembro de 2017, no curso de Enfermagem do Centro de Ciências da Saúde (CCS) da UVA, em Sobral - CE. Optou-se por essa Instituição de Ensino Superior (IES) pois o curso de Enfermagem apresenta-se no histórico da região como o primeiro de nível universitário a ser ofertado no interior do estado, caracterizando uma alínea social no processo de formação de enfermeiros para o mercado de trabalho.

O referido curso de Enfermagem possuía 355 estudantes regularmente matriculados entre o primeiro e o décimo períodos no semestre 2016.1, sendo 244 matriculados nos módulos de Atenção Básica à Saúde (ABS) I a VII (do 1으o 7 으 periodo) e 111 matriculados nos Internatos em Enfermagem I a III (do 80 ao 10 o período) ${ }^{(3)}$. A escolha dos estudantes matriculados nos referidos módulos deu-se por estes serem obrigatórios e pré-requisitos no decorrer dos 10 semestres do curso. Pretendeu-se, neste estudo, que a amostra coincidisse com a população. No entanto, foram inclusos no estudo os estudantes que assinaram o Termo de Consentimento 
Livre e Esclarecido (TCLE) e estavam presentes em sala de aula no momento da coleta de dados. Assim, do total de 355 acadêmicos regularmente matriculados, 276 (77,7\%) estudantes compuseram a amostra desta pesquisa.

A coleta dos dados se deu por meio da utilização de um questionário, com perguntas abertas e fechadas, contendo variáveis sóciodemográficas, que são: sexo, idade, estado civil, raça/cor e renda familiar. Os dados foram coletados a partir da plataforma Google Forms ${ }^{\circledR}$, website de elaboração de questionários eletrônicos. O questionário desta pesquisa foi adaptado do utilizado na pesquisa "Perfil da Enfermagem no Brasil" da FIOCRUZ/COFEn ${ }^{(4)}$. Antes da aplicação dos instrumentos foi realizado um pré-teste, com um acadêmico de cada período letivo. O referido questionário foi enviado aos acadêmicos de Enfermagem após a coleta do endereço eletrônico em sala de aula, durante o horário normal dos módulos de ABS e encontros dos internatos. Passado o período de coleta dos dados, que ocorreu durante os meses de março a abril de 2016, estes foram sistematizados para posterior análise, em planilhas do Excel® e organizados de forma tabular, e analisados estatisticamente, segundo frequências absolutas e percentuais.

A pesquisa foi realizada com a permissão da Coordenação do Curso de Enfermagem da referida IES, por meio do envio da Carta de Anuência e o projeto da pesquisa foi submetido ao Comitê de Ética em Pesquisa (CEP), sendo este aprovado por meio do protocolo de número: 50965515.8.0000.5053.

\section{RESULTADOS}

A descrição do perfil dos acadêmicos de Enfermagem mostra-se necessária/importante, ao passo que possibilita refletir sobre as características, fragilidades e o contexto político, social e econômico desse grupo social, para que se possa inferir na formação (projeto didático-pedagógico) e intervir nas necessidades sociais e fragilidades que emergem no público estudado (Tabela).

Tabela - Perfil sociodemográfico dos acadêmicos de Enfermagem da UVA. Sobral - Ceará, 2017.

$\begin{array}{lrl}\text { VARIÁVEIS } & \mathbf{N} & \% \\ \text { Sexo } & 217 & 78,6 \\ \text { Feminino } & 59 & 21,4 \\ \text { Masculino } & 276 & 100,0 \\ \text { Total } & & \\ \text { Faixa Etária (anos) } & 87 & 31,5 \\ <20 & 156 & 56,5 \\ 20 \text { a } 24 & 27 & 9,8 \\ 25 \text { a } 29 & 6 & 2,2 \\ 30 \text { e mais } & \mathbf{2 7 6} & \mathbf{1 0 0 , 0} \\ \text { Total } & & \end{array}$

Continuação.

\begin{tabular}{lrl} 
VARIÁVEIS & N & $\%$ \\
Estado Civil & 248 & 89,9 \\
Solteiro/a & 20 & 7,2 \\
Casado/a & 5 & 1,8 \\
União consensual/estável & 3 & 1,1 \\
Divorciado/a & $\mathbf{2 7 6}$ & $\mathbf{1 0 0 , 0}$ \\
Total & & \\
Cor/Raça & 158 & 57,2 \\
Parda & 93 & 33,7 \\
Branca & 15 & 5,4 \\
Preta & 10 & 3,7 \\
Amarela & $\mathbf{2 7 6}$ & 100,0 \\
Total & & \\
Renda Familiar(Salário Minimo-SM) & 30 & 10,9 \\
<l SM & 85 & 30,8 \\
1 SM & 68 & 24,6 \\
2 SM & 54 & 19,6 \\
3 SM & 39 & 14,1 \\
4 e mais SM & $\mathbf{2 7 6}$ & $\mathbf{1 0 0 , 0}$ \\
\hline TOTAL & & \\
\hline
\end{tabular}

\section{DISCUSSÃO}

O estudo apresenta a predominância de acadêmicos de Enfermagem do sexo feminino (217 - 78,6\%). Conforme o Instituto Nacional de Estudos e Pesquisas Educacionais Anísio Teixeira (INEP) do Ministério da Educação, o Censo da Educação Superior de 2009 revelou uma predominância de estudantes do sexo feminino. Mostrando que as mulheres correspondem a 55,1\% do número total de matrículas e a $58,8 \%$ do número total de concluintes ${ }^{(5)}$.

Entre os trabalhadores da Enfermagem mundial e brasileira sempre foi muito presente a figura feminina. As ações historicamente desenvolvidas pelas enfermeiras tiveram bem próximas àquelas dos cuidados maternos, de enfermos nos lares, de sujeitos em situação de risco ou vulnerabilidade ${ }^{(6)}$

No entanto, o presente estudo revela que, $21,4 \%$ dos acadêmicos de Enfermagem são homens. Apesar do número de mulheres ainda ser bem maior, nota-se um aumento no número de acadêmicos do sexo masculino. A participação crescente de homens no setor saúde, principalmente na Enfermagem, nos leva a afirmar o surgimento de uma nova tendência nesse campo, o caminhar para a igualdade de gênero da categoria. Na pesquisa do "Perfil da Enfermagem Brasileira" foi identificado que $14,4 \%$ dos profissionais são homens $^{(7)}$.

A Enfermagem vem evoluindo e conquistando novas áreas e espaços de atuação dentro dos campos da gestão, ensino, pesquisa, movimento político-profissional, dentre 
outros, revelando uma visão mais ampliada do conceito do profissional enfermeiro. O interesse de homens pelo trabalho de enfermeiro é recente, do início da década de 1990, e vem se firmando, aos poucos vai desmitificando o histórico da profissão exclusiva para mulheres ${ }^{(8)}$.

Com relação à idade dos acadêmicos, percebe-se o ingresso destes cada vez mais jovens. Vários podem ser os fatores relacionados, desde as exigências do atual mercado econômico, a maior possiblidade de ingresso mais cedo na universidade, refletindo uma melhoria de acesso à formação universitária e, consequentemente, formação profissional qualificada. Além disso, o ingresso cada vez mais cedo na universidade pode estar atrelado a uma possivel cobrança familiar e social em iniciar uma graduação imediatamente após o ensino médio.

No que concerne à faixa etária, há uma predominância de adultos jovens, 20 a 24 anos (156 - 56,5\%), seguida de adolescentes, dos menores de 20 anos (87 - 31,5\%). A menor idade encontrada neste estudo foi de 16 anos. Contudo, corroboramos com os resultados do "Perfil da Enfermagem no Brasil"(7,8), que a Enfermagem está em processo de rejuvenescimento.

Os sujeitos deste estudo, em sua maioria (88\% - 243), estão na primeira fase da vida profissional, que é denominada “Início da vida profissional". É nessa etapa em que os jovens ainda estão sem definição clara do campo de atuação e vivem o sonho da profissão, de uma vida profissional promissora, mas também da indecisão e busca da inserção no mercado de trabalho(7).

O fato de a maioria dos acadêmicos serem adolescentes e adultos jovens nos faz refletir acerca das metodologias utilizadas durante o processo de ensino-aprendizagem e a qualidade da formação, já que o tratar com esse público requer habilidades e competências especificas, com o uso de metodologias ativas, em que o acadêmico é estimulado ao pensamento crítico, e é o principal agente do seu próprio aprendizado.

Quanto ao estado civil, há a predominância de solteiros, 89,9\% (248). Os dados coincidem com estudos realizados em São Paulo e no Rio de Janeiro(9,10). Os autores desses estudos levantam a hipótese dessa variável, possivelmente, receber influência da grade curricular do curso exigir dedicação integral, por isso ter mais estudantes com estado civil solteiro. A irregularidade de distribuição dos estágios dos módulos, hora manhã, hora tarde e por vezes noite, também dificulta os compromissos familiares para os casados e/ou que necessitam trabalhar.

No que concerne à variável raça/cor, utilizou-se a nomenclatura estabelecida pelo Instituto Brasileiro de Geografia e Estatística (IBGE). Logo, 57,2\% (158) dos acadêmicos se classificaram como pardos; 33,7\% (93) como brancos; 5,4\% (15) como pretos; 3,7\% (10) amarelos. Somando pardos e pretos, o percentual chega a $60,9 \%$, tornando o grupo negro o mais expressivo nessa variável. Na pesquisa do "Perfil da Enfermagem no Brasil", predominou (57,9\%) os que se consideram brancos ${ }^{(7)}$.

Sabe-se que em 2012 foi sancionada a Lei Federal no 12.711 , que dispõe sobre o sistema de cotas para as IES, estratégia que visa tornar mais justo o acesso da população à graduação; porém, o sistema se estende apenas às instituições federais (BRASIL, 2012b). A UVA, por ser uma universidade estadual, não possui sistema de cotas. No entanto, no dia 17 de janeiro de 2017, foi sancionada a Lei Estadual no 16.197, que institui o sistema de cotas para as IES públicas no estado do Ceará(11).

No tocante à renda familiar dos acadêmicos, 10,9\% (30) com renda menor que um salário mínimo (SM), 30,8\% (85) com renda de um SM, e 41,7\%, (115) dos acadêmicos possuem renda familiar mensal de até um SM nacional. Os dados mostram um número significativo de estudantes abaixo da linha da pobreza, vivendo com menos de um SM, utilizando-se de benefícios sociais do Governo Federal.

Talrenda é insuficiente para, além de supriras necessidades familiares, manter o filho acadêmico de Enfermagem em um curso integral. Esse fato associado à carga horária integral e às demandas e exigências próprias do curso, a exemplo de vestuário, livros, instrumentais etc. pode repercutir na desistência de muitos no decorrer da vida acadêmica, uma vez que este buscará renda extra e não conseguirá a dedicação que o curso exige. Aliado a isso, tem-se ainda que grande parte dos acadêmicos (189 - 68,5\%) é oriunda de diversos municípios do Noroeste cearense e até de outros estados brasileiros, que não o de sede da Universidade (Sobral), e estes e suas famílias têm que arcar com as despesas de passagens, hospedagem e alimentação, por falta de casa do estudante e restaurante universitário somente ter sido inaugurado este ano.

O estudante universitário enfrenta diversos obstáculos no decorrer da sua formação, a dificuldade financeira é, sem dúvida, uma das maiores a ser vencida. Desde a sua saída de casa, o caminho percorrido até a sala de aula, os materiais necessários para a execução das atividades acadêmicas, dentre outras, geram gastos. Apesar da existência de bolsas de apoio financeiro ofertadas pela Universidade, o número disponibilizado não supre a real necessidade de todos os estudantes.

Corroboramos com autores ao afirmarem que se faz necessária a elaboração de projetos de pesquisa, extensão e monitoria voltados para a oferta de bolsas aos ingressantes, além da implantação de alojamentos e refeitórios com alimentação gratuita, bem como a efetivação de uma política 
que defenda a meia passagem de transporte coletivo para o universitário(12). Contudo, a trajetória do estudante de Enfermagem é repleta de grandes abdicações e ansiedade, desde o momento de sua entrada na Universidade até a formatura.

\section{CONCLUSÃO}

Diante dos resultados desta pesquisa, podemos apontar alguns que merecem destaque, dentre eles: o crescimento do número de acadêmicos do sexo masculino ingressante no curso de graduação em Enfermagem; a predominância de adolescentes e adultos jovens, sugerindo um grande poder de transformação da Enfermagem, porém, repercutindo diretamente na necessidade da competência docente no tratar com esse público; atenção maior à dificuldade financeira, o que exige a implantação de mais projetos de extensão e pesquisa financiados como estratégia para a permanência do graduando em seu curso de formação.

Apresentar os dados desta pesquisa é mostrar ao corpo docente e à sociedade um retrato, possibilitando o aprimoramento da grade curricular do curso, tornando-a mais adequada à realidade e às necessidades locais, sem que interfira e prejudique na qualidade do processo ensinoaprendizagem e na qualidade da formação.

Compreende-se que são necessários mais estudos que visem a análise da temática aqui explorada, em um propósito de aprofundar o conhecimento acerca do perfil dos estudantes, a fim de aperfeiçoar e elaborar novas estratégias para a qualificação do ensino superior, ajustando a metodologia à realidade e criando caminhos para que se possa alcançar o objetivo maior que é possibilitar uma formação universitária mais transformadora e condizente com as necessidades do SUS e, consequentemente, da população.

\section{REFERÊNCIAS}

1. Brasil. Ministério da Educação e do Desporto. Diretrizes curriculares nacionais do curso de Graduação em Enfermagem. Resolução no 3, de 7 de novembro de 2001 . Institui diretrizes curriculares nacionais do curso de Graduação em Enfermagem. Diário Oficial [da União], Brasilia, 7 nov. 2001; Seção 1, p. 37. [cited 2016 Jan 20]. Available from: http:// portal.mec.gov.br/cne/arquivos/pdf/CESO3.pdf.

2. Brasil. Ministério da Educação. Sistema e-MEC. Instituições de educação superior e cursos cadastrados. 2015. [cited 2015 Out 20]. Available from: http://emec.mec.gov.br/.

3. Universidade Estadual Vale do Acaraú (UVA). Pró-Reitoria de Ensino de Graduação. Sistema UVA - Módulo Coordenador. Relatório de Alunos Matriculados por Curso e Disciplina Curso: Enfermagem - Bacharelado, Semestre 2015.2. Sobral-CE: UVA, 2015. [cited 2015 Out 28]. Available from: http://coordenador.uvanet.br/acesso_sec. php? parameterSession $=400548$ parameterChave $=$ be $46 a 073$ eadb3f6b43272233eb96312la5cbea46.

4. Fundação Oswaldo Cruz (FIOCRUZ). Conselho Federal de Enfermagem (COFEn). Perfil da Enfermagem no Brasil: Questionário. Rio de Janeiro: FIOCRUZ, 2013.

5. Brasil. Ministério da Educação. Instituto Nacional de Estudos e Pesquisas Anisio Teixeira Educacionais-INEP. Resumo técnico: censo da educação superior de 2009. Brasilia: MEC/INEP, 2010. [cited 2016 Mai 20]. Available from: http://download.inep.gov.br/download/superior/ censo/2009/resumo_tecnico_2009.pdf.

6. Ximenes Neto FRG, Mequita OMS, Leite JL, Ferreira AGN, Melo MSS, Brasil RWN. Trabalho dos enfermeiros no controle das doenças sexualmente transmissiveis: análise do perfil, necessidades e facilidades vivenciadas na Estratégia Saúde da Familia. Paraninfo Digital [internet]. 2012 [cited 2017 Ago 20]; VI(16):1/070d-12. Available from: http://www. index-f.com/para/nl6/070d.php.
7. Machado MH, Aguiar Filho W, Lacerda WF, Oliveira E, Lemos W, Wermelinge M, Vieira M, Santos MR, Souza Júnior PB, Justino E; Barbosa C. Caracteristicas gerais da Enfermagem: o perfil sociosemográfico. Enferm. Foco [internet]. 2016 [cited 2017 Mai 12]; 7(n. especial):11-17. Available from: http://revista.cofen.gov.br/index.php/enfermagem/ article/view/686/296

8. Machado M H, Wermelinger M, Vieira M, Oliveira E, Lemos W, Aguiar Filho W et al. Aspectos Gerais da Formação da Enfermagem: O Perfil da formação dos enfermeiro, técnico e auxiliares. Enferm. Foco. [internet]. 2016 [cited 2017 Mai 12]; 7(n. especial):15-34. Available from: http:// revista.portalcofen.gov.br/index.php/enfermagem/article/view/687.

9. Donati L, Alves M J, Camelo SHH. O perfil do estudante ingressante no curso de Graduação em Enfermagem de uma faculdade privada. Rev. Enferm. UERJ. [internet]. Set. 2010 [cited 2017 Jun 12]: 16(3):446-450. Available from: http://www.facenf.uerj.br/v18n3/ v18n3al9.pdf.

10. Wetterich NC, Melo MRAC. Perfil sociodemográfico do aluno do curso de graduação em enfermagem. Revista Latino-Americana de Enfermagem. [internet]. June 2007 [cited 2017 Sep 20]; 15(3):404-10. ISSN 1518-8345. Disponivel em: https://www.revistas.usp.br/rlae/article/view/2447/2804.

11. Ceará. Poder Executivo. Lei no 16.197, de 17 de janeiro de 2017. Diário Oficial [do Estado], Fortaleza, 18 de janeiro de 2017. [cited 2017 Mai 12]. Available from: https://www.jusbrasil.com.br/diarios/134509037/doece-18-01-2017-pg-1.

12. Souza NVDO, Penna LHG, Cunha LS, Baptista AAS, Mafra IF, Mariano DCA. Perfil socioeconômico e cultural do estudante ingressante no Curso de Graduação em Enfermagem. Rev. Enferm. UERJ. [internet]. Dez. 2013 [cited 2017 Jun 20]; 21(esp.2):718-22. Available from: http:// www.facenf.uerj.br/v2lesp2/v2le2a04.pdf. 